\title{
The Influence of Pioneer Neurons on a Growing Motor Nerve in Drosophila Requires the Neural Cell Adhesion Molecule Homolog FasciclinII
}

\author{
Natalia Sánchez-Soriano and Andreas Prokop \\ Wellcome Trust Centre for Cell Matrix Research, Faculty of Life Sciences, The University of Manchester, Manchester M13 9PT, United Kingdom
}

\begin{abstract}
The phenomenon of pioneer neurons has been known for almost a century, but so far we have little insights into mechanisms and molecules involved. Here, we study the formation of the Drosophila intersegmental motor nerve (ISN). We show that aCC/RP2 and U motor neurons grow together at the leading front of the ISN. Nevertheless, aCC/RP2 neurons are the pioneers, and U neurons are the followers, because only aCC/RP2 neurons effectively influence growth of the ISN. We also show that this influence depends on the neural cell adhesion molecule homolog FasciclinII. First, ablation of aCC/RP2 has a stronger impact on ISN growth than U ablation. Second, strong growth-influencing capabilities of aCC/RP2 are revealed with a stalling approach we used: when aCC/RP2 motor axons are stalled specifically, the entire ISN (including the U neurons) coarrests, demonstrating that aCC/RP2 neurons influence the behavior of U growth cones. In contrast, stalled U neurons do not have the same influence on other ISN motor neurons. The influence on ISN growth requires FasciclinII: targeted expression of FasciclinII in U neurons increases their influence on the ISN, whereas a FasciclinII loss-of-function background reduces ISN coarrest with stalled aCC/RP2 axons. The qualitative differences of both neuron groups are confirmed through our findings that aCC/RP2 growth cones are wider and more complex than those of $U$ neurons. However, $U$ growth cones adopt aCC/RP2like wider shapes in a FasciclinII loss-of-function background. Therefore, FasciclinII is to a degree required and sufficient for pioneerfollower interactions, but its mode of action cannot be explained merely through an equally bidirectional adhesive interaction.
\end{abstract}

Key words: pathfinding; growth cone; motor neuron; axon; ablation; filopodia

\section{Introduction}

An essential feature of nervous system development is the capability of neurons to form axonal and dendritic protrusions that elongate and form contacts with appropriate target cells, thus establishing reproducible neuronal circuits. Neuritic elongation is performed by advancing growth cones at the tips of neuronal protrusions (Harrison, 1910). Growth cones perform filopodiadependent migration regulated and orchestrated by spatially arranged mixtures of guidance cues represented by diffusible or contact-mediated molecules in the environment of the growth cone. Surface molecules and intracellular signaling properties of each individual growth cone determine whether it ignores local cues or is attracted or repelled by them, resulting in their regulated and reproducible navigation (Tessier-Lavigne and Good-

Received June 16, 2004; revised Nov. 8, 2004; accepted Nov. 10, 2004.

This work was funded by grants from the Volkswagen-Stiftung (I/75 471 and I/78 743) and the Deutsche Forschungs-Gemeinschaft (PR605/1-2) to A.P. We thank C. Schuster, the Bloomington stock center, J. B. Jaynes, L. Luo, E. Martín-Blanco, H. Oda, A. Hidalgo, and M. Hoch for sending flies; E. Buchner, U. Thomas, D. Kiehart, and M. González-Gaitán for provision of antibodies; and D. Sattelle for providing Roadl cDNA. Most of this work was performed in the Institute of Genetics of Johannes Gutenberg University (Mainz, Germany). We express our gratitude to G. M. Technau for provision of laboratory space and infrastructure at the institute and for his helpful advice. Furthermore, we are grateful to H. Hutter and M. Landgraf for helpful comments on this manuscript.

Correspondence should be addressed to Natalia Sánchez-Soriano, Wellcome Trust Centre for Cell Matrix Research, Faculty of Life Sciences, The University of Manchester, Michael Smith Building, Oxford Road, Manchester M13 9PT, UK. E-mail: NSanchez@manchester.ac.uk.

DOI:10.1523/JNEUROSCI.2377-04.2005

Copyright $\odot 2005$ Society for Neuroscience $\quad$ 0270-6474/05/250078-10\$15.00/0 man, 1996). At the cell biological level, these dynamic interactions are reflected by the fact that growth cones alter their shapes in a reproducible manner (Tosney and Landmesser, 1985; Murray et al., 1998).

Whereas growth cones pioneering a future fiber tract have to read and interpret environmental cues in their full complexity, "the fibers which develop later follow, in the main, the paths laid down by the pioneers," as first described by R. G. Harrison almost 100 years ago (Harrison, 1910). Experiments testing the requirement of pioneer neurons for correct growth of follower neurons led to three different scenarios: (1) pioneer neurons appear unnecessary for guidance of later outgrowing neurons (Keshishian and Bentley, 1983; Eisen et al., 1990); (2) pioneer neurons facilitate but are unnecessary for pathfinding by later outgrowing neurons (Pike et al., 1992; Lin et al., 1995; Bak and Fraser, 2003); (3) pioneer neurons appear to be absolutely required for normal pathfinding by later outgrowing neurons (Raper et al., 1984; Kuwada, 1986; Klose and Bentley, 1989; Ghosh et al., 1990; Pike et al., 1992; Hidalgo and Brand, 1997; Williams and Shepherd, 2002). These observations were mostly based on studies of follower neurons under conditions in which their pioneer neurons had been ablated. However, cell ablations also remove cues displayed by the ablated pioneer neurons that are likely to influence the growth cones of followers. Consistent with this idea, filopodia of follower growth cones have in some contexts been shown to be closely attached to pioneer axons but changed shape after pioneer 
ablation (Kim et al., 1991; Bak and Fraser, 2003). Which are the molecules required for such influence on follower growth cones?

To approach this question, we either ablated or stalled identified motor axons. The cellular system used here is the developing intersegmental motor nerve (ISN) of Drosophila embryos. In the first step, we clarify some aspects left open by former studies (Jacobs and Goodman, 1989; Lin et al., 1995) and show aCC/RP2 neurons to be functional pioneer neurons of the ISN. In the second step, we apply the stalling strategy and show that influences of aCC/RP2 neurons on ISN growth are much stronger than suggested by ablation experiments. Using the stalling approach, we can show that FasciclinII is required for the influence of aCC/ RP2 on ISN growth.

\section{Materials and Methods}

Fly stocks. Fly strains used in our studies are fasciclinII $I^{\text {eb112 }}$ mutant alleles (Grenningloh et al., 1991), elav-Gal4 (Bloomington stock center, Bloomington, IN) (Luo et al., 1994), different Gal4 lines carrying promoter fragments of the even skipped gene ( $R N 2-G a l 4^{D+O}$, second chromosome; RN2-Gal4 ${ }^{E}$, third chromosome; eve-Gal4 ${ }^{R R K}$, third chromosome; U/CQGal4, third chromosome; courtesy of J. B. Jaynes, Thomas Jefferson University, Philadelphia, PA) (Fujioka et al., 1999, 2003), MzVum-Gal4 (Landgraf et al., 2003), UAS-mCD8-green fluorescent protein [UAS$m C D 8$-GFP; second and third chromosome; courtesy of L. Luo (Stanford University, Stanford, CA) and the Bloomington stock center] (Lee and Luo, 1999), different fly strains carrying UAS-coupled genes such as dominant-negative Rho-GTPase UAS-DRac1 ${ }^{N 17}$ (courtesy of E. MartínBlanco, CSIC, Barcelona, Spain) (Luo et al., 1994), UAS-GFP-actin ${ }^{2-2}$ (courtesy of H. Oda, JT Biohistory Research Hall, Osaka, Japan) (Verkhusha et al., 1999), UAS-ricinA (second chromosome; courtesy of A. Hidalgo, School of Biosciences, Birmingham, UK) (Hidalgo et al., 1995), and UAS-Notch ${ }^{I C N}$ (courtesy of M. Hoch, Universitat Bonn, Bonn, Germany) (Kidd et al., 1998).

Immunohistochemistry. Embryos were dissected in external bath solution following standard protocols (Broadie, 2000). Dissected embryos were fixed 30-60 min in 4\% paraformaldehyde in $0.05 \mathrm{~m}$ phosphate buffer, $\mathrm{pH} 7-7.2$, and washed for $1 \mathrm{hr}$ in PBS with $0.1 \%$ Triton X-100 (PBT). Incubation with antibodies was performed in PBT without blocking reagents. We used antibodies raised against presynaptic protein synapsin (mouse, 1:10; courtesy of E. Buchner, Universitaet Wuerzburg, Wuerzburg, Germany) (Klagges et al., 1996), the transmembrane domain protein CD8 (rat, 1:10; Caltag Laboratories, Burlingame, CA), the cytoplasmic domain of the cell adhesion molecule FasciclinII (1D4 supernatant, mouse, 1:10; Developmental Studies Hybridoma Bank, Iowa City, IA) (Vactor et al., 1993), the predominantly postsynaptic structural protein Discs large (Dlg) (rabbit, 1:1000; courtesy of U. Thomas, Institute for Neurobiology, Magdeburg, Germany) (Woods and Bryant, 1991; Lahey et al., 1994), the muscular protein myosin heavy chain (rabbit, 1:1000; courtesy of D. Kiehart, Duke University, Durham, NC) (Kiehart and Feghali, 1986), fluorochrome-coupled horseradish peroxidase (HRP; 1:200; Cappel, Cochranville, PA) (Jan and Jan, 1982), anti-GFP (goat, 1:1000; courtesy of M. González-Gaitán, Max-Planck-Institut für Molekulare Zellbiologie und Genetik, Dresden, Germany), and fluorochrome-coupled secondary antibodies (dilution 1:200; Jackson ImmunoResearch, West Grove, PA).

Generation of UAS-Rdl transgenic flies. A BglII-NotI fragment of the pNB40 vector, containing the Rdl cDNA NB14.1 (kindly obtained from D. Sattelle, Department of Human Anatomy and Genetics, University of Oxford, Oxford, UK) (ffrench-Constant, 1993) was cloned into a BglIINotI-digested pP(UAST) vector (Brand and Perrimon, 1993). Transformation of white mutant flies with the vector containing the UAS-Rdl gene was performed as described previously (Spradling and Rubin, 1982). Of the resulting fly stocks, a first (UAS-Rdl ${ }^{8.2}$ ) and third chromosomal insertion $\left(U A S-R d l^{1.4}\right)$ were used here.

Growth cone measurements. To guarantee unbiased documentation, the genotype of specimens was disguised when drawing the shapes of growth cones labeled by targeted expression of actin-GFP. The growth cone area and perimeter were determined using Analysis software (Soft Imaging Systems). Statistical analyses were performed using Sigma Stat software (SPSS, Chicago, IL). Data are given as median (the centermost value of the data set; i.e., at 50\%) and the interquartile range (IQR) referring to the values at 25 and $75 \%$ of the data set (IQR: $25 \%$; $75 \%$ ) is also indicated (see Fig. 6). Data were subjected to Mann-Whitney rank sum tests to compare groups. Differences were highly significant if $p<0.001$.

\section{Results}

\section{A refined cellular model to study a developing nerve}

To investigate the role of Drosophila pioneer motor neurons for the establishment of motor neuronal nerves, we focus our analyses on the most dorsal muscle area and its innervation through the ISN (Thomas et al., 1984) for several reasons. First, it is the area farthest away from the CNS requiring long-distance navigation of the ISN axons. Such growth is very likely to involve interaxonal communication as an essential regulatory feature. Second, the muscle and innervation pattern is simpler in the dorsal region than in the ventral muscle field (Bate, 1993), facilitating analysis of phenotypes. Third, this is the only area in the embryo in which all innervating neurons have been identified and can be genetically manipulated using available Gal4 lines (see Fig. 1 for details and nomenclature): in more detail, per hemisegment one RP2 and three ventral unpaired median (VUM) neurons have branches on most, if not all, muscles in the dorsal muscle field (Landgraf et al., 2003), one aCC motor neuron innervates muscle DA1, and three different $U$ motor neurons establish contacts on DO1, DO2, and DA3, respectively (Landgraf et al., 1999) (a fourth $U$ neuron that terminates on LL1 muscles is not considered here). Several Gal4-driver lines can be used to target UAScoupled genes to these neurons for their visualization and/or manipulation (Fig. 1) [for details on the Gal4/UAS-strategy, see Duffy (2002)]: RN2-Gal4 directs Gal4 expression to aCC and RP2 neurons (Fujioka et al., 2003), U/CQ-Gal4 to the U neurons (Fujioka et al., 2003), and MzVum-Gal4 to the VUM neurons (Landgraf et al., 2003). Apart from the single axons of the ISN, the entire nerve or all of its terminals can also be labeled to monitor its length. To this end, either neuronal surface markers such as FasciclinII or HRP or synaptic markers such as synapsin or Dlg can be used.

\section{aCC/RP2 and $U$ motor neurons grow at the leading edge of the forming ISN}

Former ultrastructural and ablation studies suggest that aCC motor neurons might perform pioneer functions during ISN establishment. However, as detailed below, this aspect was never fully resolved, mainly because of technical limitations. We therefore made use of the refined tools described above to complement former studies and clarify the roles of the different neuron groups during ISN formation.

Previous ultrastructural studies revealed that aCC is the first neuron of the ISN to grow out, thus defining it as the pioneer neuron of this motor nerve (Jacobs and Goodman, 1989). However, these studies were restricted to aCC growth in the CNS (i.e., the very early stage of ISN development). Therefore, we extended on these studies and compared the positions of axonal tips of the three different neuronal subpopulations (labeled with the UAS$m C D 8-G F P$ or UAS-GFP-actin ${ }^{2-2}$ reporters) relative to the entire ISN length (visualized with anti-FasciclinII) at early and later stages of development. Our studies revealed that the RN2-Gal4-positive neurons ( $\mathrm{aCC}$ and/or RP2) grow mostly at the leading front of the developing ISN (Fig. 2, top row). In contrast, the growth cones of VUM axons keep considerably behind aCC/RP2 at all developmen- 
tal stages investigated (Fig. 2, bottom row), discarding them as pioneers. The U neurons (visualized via U/CQ-Gal4) are relatively delayed at early stages of ISN formation when they begin to leave the CNS (Fig. $2 E, E^{\prime}$ ), navigating behind the axonal tips of aCC/RP2 [in agreement with Jacobs and Goodman (1989)]. However, once the U neurons join the ISN and navigate in the periphery, axonal tips of this neuron population reach the most distal end of the growing ISN in 70\% of cases ( $n=43$ hemisegments from $16 \mathrm{em}$ bryos) (Fig. $2 F-H^{\prime}$ ). Thus, although aCC and/or RP2 neurons are leading initially, $U$ neurons catch up and stay at the nerve front during later ISN development. Therefore, if defining a pioneer neuron merely by its position at the leading edge of a growing nerve, not only aCC/RP2 but also U neurons would have to be considered pioneers in most ISNs (note that in grasshoppers, $\mathrm{U}$ neurons but not aCC seem to be the pioneers) (DuLac et al., 1986).

\section{Ablation studies suggest a pioneer role for aCC/RP2 but not $U$ neurons}

To uncover potential functions of leading neurons during the formation of an entire nerve, ablation studies can be used. We performed ablation studies using RN2Gal4-driven expression of the cytotoxin ricinA, a tool that was used successfully to test neuronal pioneer functions in the Drosophila CNS (Hidalgo and Brand, 1997).

To test for ablation efficiency, we analyzed the patterns of three independent cell markers in control embryos and embryos with RN2-Gal4-induced ricinA expression (Fig. $3 A-D^{\prime}$ ). Already at the very early stage of axonal growth (early stage 13), marker expression was affected severely but cell specifically in ricinA-expressing aCC/RP2 neurons (Fig. $3 A-D^{\prime}$, open vs white symbols). This indicates that ricinA induced neuronal degeneration sets in at very early stages, strongly suggesting that manipulated aCC/RP2 neurons do not grow motor axons. Effects on ISN morphology resulting from specific aCC/RP2 ablation were analyzed at late stage 17 (i.e., the time of hatch). Although dissections at this stage are difficult, they enable us to analyze experimental impacts on matured neuronal connections, whereas analyses at earlier stages may be hampered by transient abnormalities or developmental delays. In the case of ricinAinduced aCC/RP2 ablations, growth of the entire ISN is affected in $26 \%$ of cases, as revealed by anti-FasciclinII staining at late stage 17 ( $n=42$ hemisegments) (Fig. 3J, white arrowhead). The defects primarily consist of premature stalling of the nerve mostly at the level of muscles DO2/DA2. This indicates that, at rather low frequency, the nonmanipulated U and VUM neurons fail to reach their dorsal target muscles as a consequence of the absence of aCC/RP2. These data show the same tendency as former ablation studies in Drosophila in which another promoter construct and cytotoxin were used (12\% of nerves were affected in the absence of aCC at stage 16) (Lin et al., 1995).

Might this low impact of aCC/RP2 ablations be attributable to the fact that $U$ neurons share pioneer functions with aCC at the leading ISN front? Because this possibility has never been tested, we ablated U neurons using U/CQ-Gal4-driven ricinA. Although $\mathrm{U}$ neurons are as severely affected at early stage 13 as aCC/RP2 in the above experiments (Fig. $3 E-H^{\prime}$, open symbols), their absence has an impact on the dorsal outgrowth of the ISN in only $2.9 \%$ of cases ( $n=204$ hemisegments) (Fig. $3 K$ ), thus at much lower frequency than in the case of aCC/RP2 ablation.

Together, these data show that ISN growth does not essentially depend on the presence of aCC/RP2 or U neurons. However, although $\mathrm{U}$ neurons and aCC/RP2 neurons both grow at the leading edge of the ISN, they are different in their abilities: aCC and/or RP2 neurons play a more important role in ISN development than $\mathrm{U}$ neurons.

\section{aCC/RP2 neurons have a stronger influence on ISN development than their ablation reveals}

As explained in the Introduction, effects observed in ablation experiments do not necessarily reflect the full influence that pioneers may have on follower neurons during normal development. If pioneer neurons and the potential cues they display are ablated, pathfinding cues from other sources may gain in importance for the guidance of follower neurons and compensate for the loss of the pioneers.

To test this possibility for aCC/RP2, we used a stalling approach based on targeted expression of the ionotropic GABA receptor Rdl (UAS-Rdl). When Rdl was targeted to aCC/RP2, their axons stalled mostly in the dorsolateral muscle field before reaching their most dorsal target muscles (in 70\% of 134 hemisegments) (Figs. $1,4 A^{\prime}$ ). Such stall was not caused by neuronal 




Figure 2. U and aCC/RP2 motor axons grow at the leading edge of the ISN. The ISN was analyzed at different time points $(A, E$, and $I$, stage $13 ; B, F$, and $J$, stage $14 ; C, D, G, H$, and $K$, stage 15). Preparations are labeled with anti-Fasciclinll (Fasll; magenta throughout); in addition, the different motor neurons (respective Gal4 lines indicated in boxes on the left) display targeted expression of mCD8-GFP or GFP-actin (green, as indicated at the top). Picture pairs show mCD8-GFP alone (no prime) or doublelabeled with Fasciclinll (prime). Nerve tips of the entire ISN are indicated by open arrowheads, tips of $m$ CD8-labeled motor axons are indicated by white arrowheads, and positions of white arrowheads are shown as close-ups. Note that aCC/RP2 neurons grow at the leading edge of the ISN at all analyzed stages $\left(A^{\prime}-C^{\prime}\right)$, U neurons are delayed initially $\left(E^{\prime}\right)$ but grow at the leading edge later on $\left(F^{\prime}, G^{\prime}\right)$, and VUM neurons stay behind at all developmental stages ( $I^{\prime}-K^{\prime} ; 100 \%$ of cases; $n=45$ from 10 embryos). Because mCD8-GFP often fails to show details such as filopodia, experiments were repeated with GFP-actin expression that likewise reveal that aCC/RP2 filopodia can be seen at the very tip of the Fasciclinll-labeled ISN in $91 \%$ of cases ( $D ; n=22$ hemisegments of 8 embryos); $U$ axons grow at the ISN tip in $70 \%$ of cases $(H ; n=45$ hemisegments of 10 embryos). A second outgrowing nerve (bent open arrows) represents the substantia nigra pars compacta as suggested by the presence of MzVUM-Gal4 targeted axons (white curved arrow in $K, K^{\prime}$ ) (Landgraf et al., 2003). Scale bar, $20 \mu \mathrm{m}$. of DRac1 ${ }^{\mathrm{N} 17}$ (dominant-negative form of the Rho-like GTPase) (Luo et al., 1994) and Notch ${ }^{\text {ICD }}$ (activated form of the transmembrane receptor Notch) (Kidd et al., 1998) equally induced stalls of aCC/RP2 and led to frequent coarrest of the complete ISN (Figs. $1,4 D, E)\left(D R a c 1{ }^{\mathrm{N} 17}, n=\right.$ 197 analyzed hemisegments, $15.2 \%$ of aCC/RP2 stall, $90 \%$ of these cause coarrest; Notch ${ }^{\text {ICD }}, n=105,63.8 \%$ stall, $71 \%$ coarrest). Thus, three very different forms of targeted manipulation used to stall aCC/ RP2 axons reveal the same quality of these neurons (i.e., the ability to cause coarrest of other motor axons in the developing ISN). To simplify experimental work, the following experiments were restricted to the use of UAS-Rdl.

To find out whether other neurons of the ISN would differ from aCC/RP2 with respect to their growth-influencing properties, Rdl was targeted by U/CQ-Gal4 or MzVum-Gal4. Like aCC/RP2 axons, axons of $U$ and VUM neurons also stalled after Rdl expression, respectively. However, these stalls had only a mild effect or had no effect on the rest of the ISN neurons (the ISN coarrests with stalling U neurons in $10 \%$ of cases and with stalling VUMs in $0 \%$ of cases; $n=128$ and 58, respectively) (Figs. $\left.1,4 B, B^{\prime}, C, C^{\prime}\right)$.

Thus, only axons of aCC/RP2 but not axons of $U$ neurons and VUMs have a strong impact on ISN growth (shown by stalling), although aCC/RP2 neurons are not absolutely required for ISN formation (shown by ablation). Therefore, we refer from now on to aCC/RP2 as the pioneers and to U neurons and VUMs as follower neurons of the ISN. This definition refers to their influential properties rather than to their positions in the growing ISN. Consistent with this assignment is our finding that aCC/RP2 and $U$ neurons display different growth cone shapes typical of pioneer and follower neurons (see below) (Bak and Fraser, 2003). debility or degeneration, because aCC/RP2 motor neurons expressing $\mathrm{Rdl}$ at late larval stages ( $3 \mathrm{~d}$ later using eve-Gal4 ${ }^{R R K}$ ) looked relatively normal (axonal and dendritic processes of correct size persisted, and neuromuscular terminals displayed presynaptic markers and boutons; data not shown). We next analyzed the impact that stalling of aCC/RP2 axons might have on the growth behavior of the entire ISN. We found that stalled aCC/RP2 axons cause a coarrest of the complete ISN in $70 \%$ of cases (Fig. 4A', arrowheads), suggesting that aCC/RP2 neurons display influential properties far beyond those revealed by ablation experiments. Rdl usually influences electrical cell properties (ffrench-Constant, 1993), but its mode of action in this context is still under investigation. To confirm that defects in other ISN neurons are not a direct non-cell autonomous effect of Rdl but rather a secondary consequence of stalled aCC/RP2 axons, we used other means to stall aCC/RP2. Thus, targeted expression
The influence of pioneer motor neurons on ISN growth requires FasciclinII

Next we studied potential mechanisms involved in the influence of aCC/RP2 axons on ISN growth. One molecule that could play a role in this process is the homophilic neural cell adhesion molecule (N-CAM) FasciclinII, which is expressed on the surface of all ISN motor neurons (see above) (Grenningloh et al., 1991; Vactor et al., 1993).

One way to analyze FasciclinII function is through overexpression studies (Lin and Goodman, 1994; Davis et al., 1997). We used this approach in combination with our stalling strategy to determine whether FasciclinII has the potential to influence pioneer-follower interactions. As explained above, U neurons frequently grow at the leading front of the ISN (Fig. 2). Nevertheless, they behave as followers (Fig. 4). However, if FasciclinII was co- 
expressed together with Rdl using the U/CQ-Gal4 driver, $54 \%$ of ISNs coarrested $(n=50)$ (Fig. $\left.4 B^{\prime}, B^{\prime \prime}\right)$, whereas in control experiments, ISNs coarrested with Rdlstalled $U$ neurons in only $10 \%$ of cases ( $n=128$ hemisegments). Thus, targeted expression of FasciclinII in U motor neurons is sufficient to increase their influence on the growth of other ISN motor neurons. This result also confirms our previous findings that $\mathrm{U}$ neurons are physically placed in a location at the tip of the growing ISN from which they can potentially influence nerve growth. Thus, it is not the position of $U$ growth cones but rather other properties that make $\mathrm{U}$ neurons different from aCC/RP2 neurons. Similarly to $\mathrm{U}$ neurons, stalled aCC/RP2 axons also had a larger influence on follower neurons when FasciclinII was coexpressed with Rdl using RN2-Gal4 (Rdl alone 70\%; Rdl plus FasciclinII 97\%) (Fig. 1).

Does endogenous FasciclinII also play a role in pioneer-follower interaction? Absence of endogenous protein in fasciclin$I I^{e b 112}$ mutant embryos causes very subtle motor neuronal growth phenotypes that do not allow any functional statement (data not shown; see also Lin and Goodman, 1994; Schuster et al., 1996). However, we speculated that if endogenous FasciclinII is involved in pioneer-follower interactions, lack of FasciclinII should lead to a reduction of ISN coarrest with stalled aCC/RP2 axons. We found that ISN neurons were coarrested in fasciclinII ${ }^{\text {eb112 }} \mathrm{mu}$ tant background in only $36 \%$ of cases $(n=$ 50 hemisegments) compared with $70 \%$ in wild-type background ( $n=74$ hemisegments) (Fig. $4 A^{\prime}, A^{\prime \prime}$ ). Thus, removal of FasciclinII causes a severe suppression of the aCC/RP2-induced phenotype and significantly restores the ability of follower neurons to grow to their natural length and targets. We take this as a strong indication that endogenous FasciclinII is required for the influence of pioneers on ISN growth.

So far, in the context of motor neuronal outgrowth, endogenous FasciclinII has been seen merely as a homophilic cell adhesion molecule that mediates fasciculation. The aCC/RP2 and U neurons all express FasciclinII endogenously (Vactor et al., 1993) and mostly grow together at the leading front of the ISN. Nevertheless, they show asymmetric influential properties (i.e., aCC/RP2 neurons behave as pioneers and $U$ neurons behave as followers). This asymmetry is unlikely to represent the mere result of homophilic adhesion of endogenous FasciclinII between the surfaces of pioneer and follower neurons. To compare the influential capabilities of aCC/RP2 and U neurons, we analyzed in greater detail the
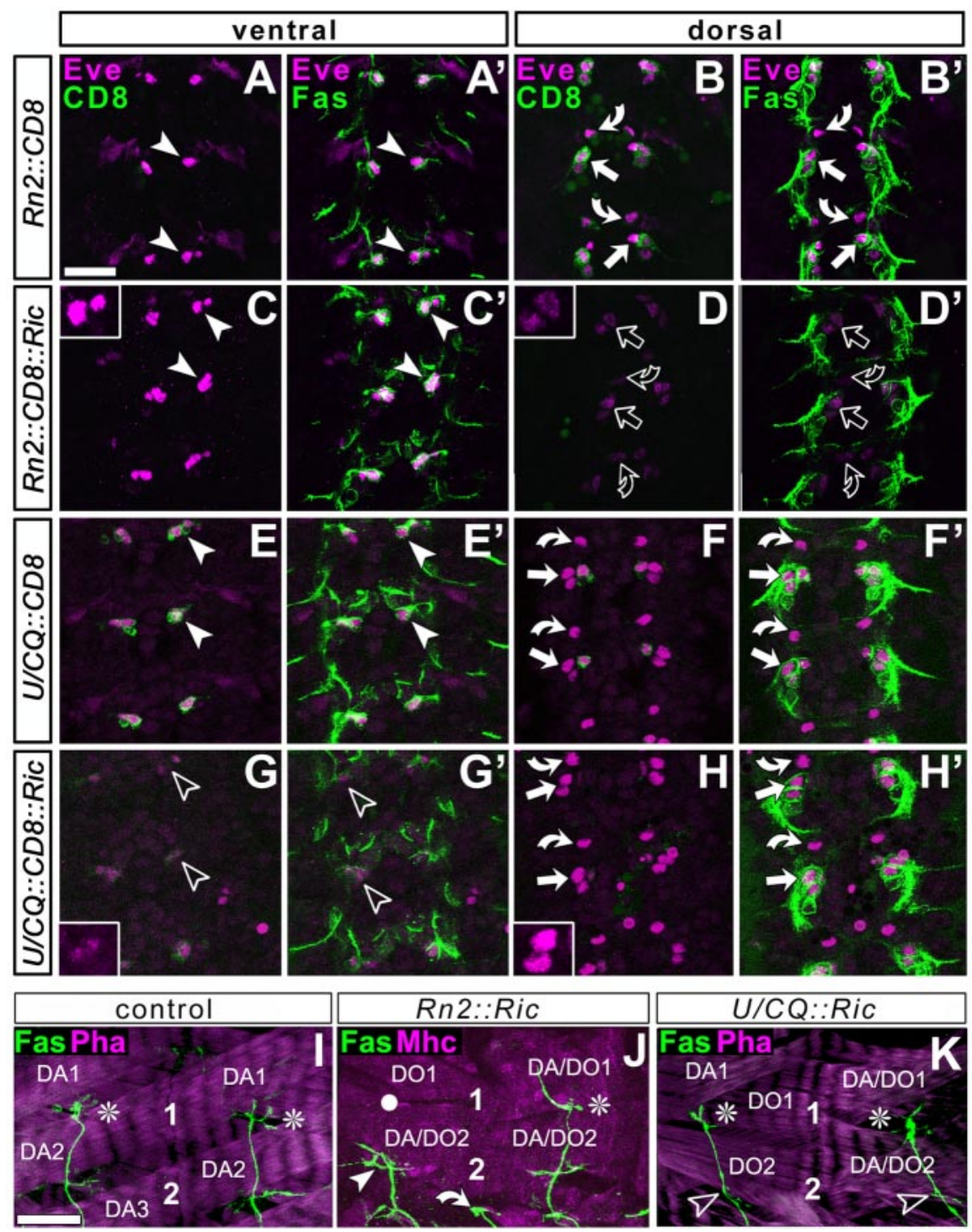

Figure 3. Ablation studies using targeted expression of ricinA. The CNS at embryonic stage $13\left(A-H^{\prime}\right)$ or dorsal muscle fields at late stage 17 ( $I-K$ ) are labeled as indicated at the top left: CD8, Gal4-induced mCD8-GFP (green in first and third column); Eve, the transcription factor Even-skipped (magenta in $A-H^{\prime}$ ); Fas, Fasciclinll (green in second and fourth column and bottom row); Mhc, the muscle marker myosin heavy chain (magenta in J); Pha, actin labeled with phalloidin (magenta in I, K). $A-H^{\prime}$, Views of the horizontal plane of an abdominal CNS (anterior up) at embryonic stage 13 (the early motor axonal growth phase) visualized at ventral (left two columns) or dorsal (right two columns) levels, respectively, as indicated in the boxes at the top. Cell bodies of ventral U neurons (arrowheads), dorsal aCCs (straight arrows), and dorsal RP2s (curved arrows) were visualized with three independent markers: Fasciclinll and Even-skipped are expressed in all of these neurons (Grenningloh et al., 1991; Broadus et al., 1995); mCD8-GFP is expressed only in those neurons targeted by the respective Gal4 line (genetic constellation indicated on left side). RN2-Gal4 targets CD8 expression to dorsal aCC/pCC and RP2 neurons (B) but not to ventral U neurons $(A)$; U/CQ-Gal4 mediated expression occurs in ventral $U$ neurons $(E)$ but not in aCC/pCC and RP2 neurons $(F)$. If these Gal4 lines are used to coexpress ricinA (Ric) with CD8, expression of all three markers is severely affected, but only in the targeted neurons (open symbols in $D, D^{\prime}$ and $G, G^{\prime}$; see especially the granular and weak occurrence of Eve; insets); furthermore, the Fasciclinll-labeled motor nerve is much thinner, especially after ablation of the larger group of $U$ neurons (data not shown). $I-K$, Dorsal muscle fields of two consecutive hemisegments, respectively, at the end of embryogenesis in control animals or specimens with neuron-specific expression of ricinA. 1 and 2 indicate motor neuronal terminals on DA1/D01 and DA2/D02 muscles, respectively (some muscles indicated for orientation; compare Fig. 1). In controls and many cases of ricinA-ablated neurons, the ISN (shown with Fasciclinll, green) grows to full length (white asterisks); in 26\% of cases in which aCC/RP2 are ablated, ISNs stall in dorsolateral areas (white arrowhead in J; white circles indicate noninnervated muscles), whereas only $2.9 \%$ of ISNs stall in the case of $U$ ablation (K, open arrowheads indicate absence of U terminals on DA/D02 muscles); curved arrow indicates transverse nerve in J. Scale bar: (in $A) A-H^{\prime}, 20 \mu \mathrm{m}$; (in I) $I-K, 16 \mu \mathrm{m}$.

behavior of the third group of neurons, the later-growing VUMs that are likewise FasciclinII-positive. If $U$ neurons are stalled, aCC/RP2 axons escape in $90 \%$ of cases. Thus, VUM neurons can choose to coarrest with $U$ terminals or grow on with aCC/RP2. 

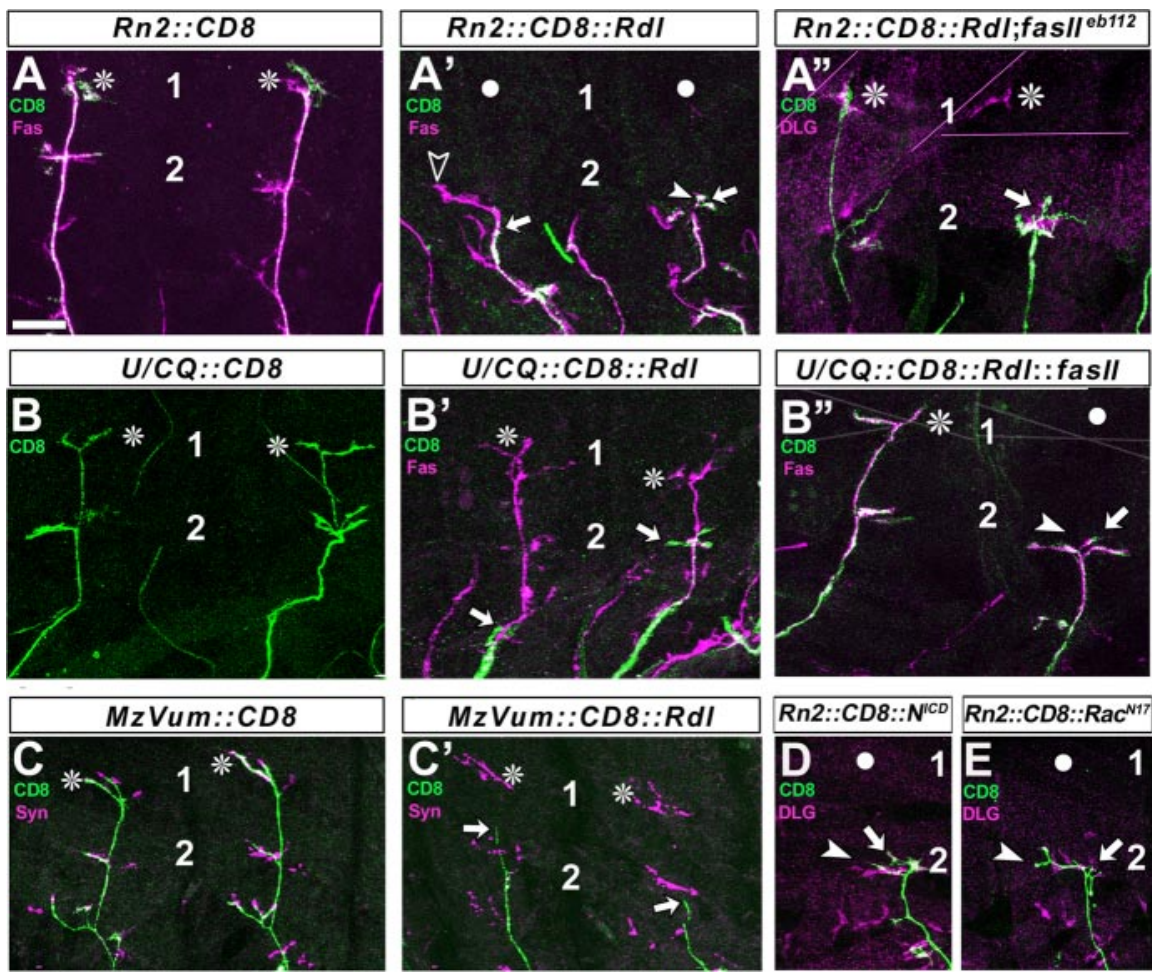

Figure 4. Axons of aCC/RP2 motor neurons can influence ISN formation. Figures show dorsal muscle fields of one or two consecutive hemisegments in late embryos (compare Fig. 1) (1 and 2 indicate position of terminals on DA1/D01 and DA/D02 muscles) with the terminals or axons of the entire ISN in magenta [visualized with Fasciclinll (Fas), synapsin (Syn), or Discs large as indicated at the top left; see Results for details] and the Rn2-Gal4, U/CQ-Gal4, or MzVum-Gal4 targeted axons in green (visualized via CD8 expression). Normally, the CD8-labeled terminals of aCC/RP2 neurons ( $A$ ), U neurons $(B)$, and VUMs ( $C$ reach most dorsal positions (asterisks). If Rdl is expressed in these neurons $\left(A^{\prime}-C^{\prime}\right)$, their axons stall frequently (straight arrows); entire ISNs tend to coarrest with stalled aCC/RP2s ( $A^{\prime}$; white circles indicate noninnervated muscles; arrowheads point at tips of ISNs, open arrowhead in $A^{\prime}$ indicates a case in which axons escaped aCC/RP2 but stalled thereafter, as in ablation experiments). ISNs mostly escape from stalled U neurons and VUMs ( $B^{\prime}, C^{\prime}$, asterisks; ISN coarrest in $R n 2:: R d I=70 \% ; U / C Q:: R d l=10 \% ; M z V u m:: R d l=0 \%$ ). Coarrest of ISNs with stalled aCC/RP2 motor axons is severely suppressed in fasciclin// ${ }^{e b 112}$ loss-of-function mutant background as shown for the right segment in $A^{\prime \prime}$ where the aCC/RP2 axons (green) are stalled, whereas Dlg-labeled terminals on dorsal muscles (magenta) are visible (ISN coarrest in $R n 2:: R d l ; f a s / I^{e b 112}=36 \%$ ). In contrast, ISN coarrest with stalled U neurons is severely increased, if Fasciclinll is coexpressed with Rdl (right axon in $B^{\prime \prime}$; ISN coarrest in U/CQ::Rdl::fas/l = 54\%). Targeted expression of activated Notch $\left(N^{I C D} ; D\right)$ or dominant-negative DRac1 $\left(\operatorname{RaC}^{N 17} ; E\right)$ in aCC/RP2s likewise leads to axon stall and coarrest of the ISN, confirming the findings with Rdl (see Fig. 1 for details). Scale bar, $16 \mu \mathrm{m}$.

Only an estimated number of $4.4 \%$ of VUM neurons coarrests ( $n=76$ hemisegments from 22 embryos when excluding cases in which aCC/RP2 coarrests) (Fig. 5A, $B^{\prime}$ ). If aCC/RP2 neurons are stalled, all ISN neurons including VUMs coarrest in at least $70 \%$ of cases (Fig. 1). More interesting are those $30 \%$ of cases in which aCC/RP2 axons stall but $U$ axons escape, providing VUM neurons again with a choice. We managed to identify 22 such cases, of which $27 \%$ showed coarrest of VUM neurons with aCC/RP2 and $73 \%$ showed escape (Fig. 5C, $D^{\prime}$ ). Thus, although aCC/RP2 axons were not influential enough to coarrest $\mathrm{U}$ axons in these cases, they still managed to coarrest VUM neurons at a much higher percentage than stalled $U$ neurons in the experiment above. Thus, VUM neurons are influenced more through aCC/RP2 than through $\mathrm{U}$ axons. A possible explanation would be that aCC/RP2 neurons express FasciclinII at higher levels than $U$ neurons. However, to our surprise, the levels of FasciclinII immunoreactivity (antibody against the intracellular Fasciclin2 domain) in many instances appeared rather low on freely visible surfaces of aCC/RP2 axons and growth cones, whereas $U$ neurons always showed strong immunoreactivity (Fig. 5C, $D^{\prime \prime}$ ). Therefore, the molecular modes of FasciclinII function during motor nerve

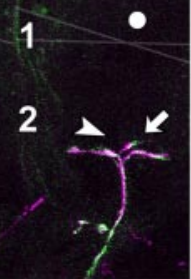

growth may involve regulations beyond mere homophilic adhesive interactions. In support of this view, a signaling function of Fasciclin2 has been demonstrated recently in Drosophila cell culture (Forni et al., 2004), as is likewise the case for its homologous proteins in other species (see Discussion for a more refined view of this matter).

Growth cone morphology supports the specific role of aCC/RP2 neurons as pioneer neurons

Having studied the pioneer and follower interactions mostly at the level of nerve growth behavior, we next extended our studies to the growth cone level. To this end, we visualized the growth cones of $U$ and aCC/RP2 motor neurons during ISN formation using targeted expression of GFP-actin. This way the growth cones of the targeted neurons were selectively labeled, also revealing their filopodial processes. We find that the pioneer growth cones of aCC/RP2 take on a wide shape with multiple scattered filopodia, whereas growth cones of $U$ neurons are narrow and appear simpler (Fig. 6A,B). To measure these differences, we determined the area and perimeter of growth cones and calculated the complexity index $\mathrm{P}^{2} / \mathrm{A}$ (square of the perimeter divided by area) (Bowie and Young, 1977). Indeed, the $\mathrm{P}^{2} / \mathrm{A}$ value of aCC/RP2 neurons is significantly higher than of $\mathrm{U}$ neurons, indicating that aCC/ RP2 growth cones are more complex (i.e., display more individually distinguishable filopodia) $(p<0.001)$ (Fig. 6D). The narrow shape of $U$ growth cones most likely reflects the fact that their filopodia are predominantly attracted by cues on the pioneer neurons. Such cues are likely to involve those that help to influence the $\mathrm{U}$ neurons to coarrest with aCC/RP2 (for example, FasciclinII).

Does FasciclinII (which is required for the influence of pioneers on ISN growth) (Figs. 1,4) mediate the simple and narrow appearance of $U$ growth cones? To address this question, we analyzed $U$ growth cones in a fasciclinII ${ }^{\text {eb112 }}$ mutant background. We found that under these conditions, $U$ growth cones take on a wide shape that is reminiscent of aCC/RP2 pioneer growth cones. Measurements of the $\mathrm{P}^{2} / \mathrm{A}$ complexity index clearly support this observation (Fig. $6 C^{\prime}, D$ ). The fact that $\mathrm{U}$ growth cones acquire pioneer-like shapes provides a good explanation for the finding that follower neurons more frequently escape stalled aCC/RP2 axons in a fasciclinII $I^{\text {eb112 }}$ mutant background, but also for their ability to grow out to full length after aCC/RP2 ablation (Fig. 3J).

Together, our observations on growth cones are consistent with our findings that aCC/RP2 neurons represent pioneers of the ISN that influence their follower neurons via molecular pathways involving FasciclinII. The fact that aCC/RP2 growth cones behave differently from $\mathrm{U}$ growth cones strongly supports the view that their pathfinding and growth-influencing properties are not the same. 


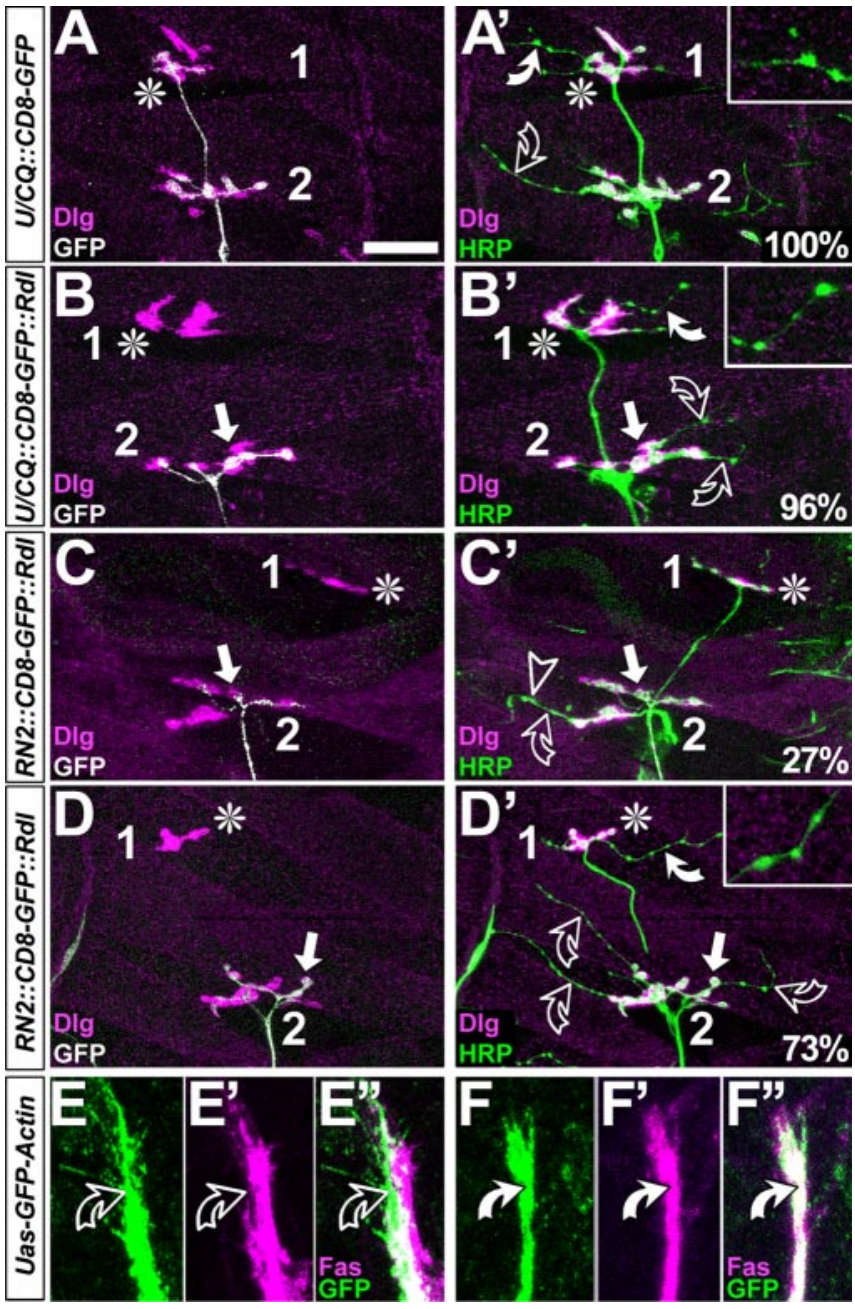

Figure 5. Fasciclinll might not act merely as a homophilic adhesion factor. $A-D^{\prime}$, Dorsal muscle fields of embryos at the late first and early second instar larval stage; symbols are as in Figure 4 and stainings are indicated at the bottom left. In control hemisegments, CD8-GFPlabeled $U$ neurons grow to the most dorsal muscle (asterisk in $A$ ), as does the entire nerve (HRP in $A^{\prime}$ ). Whereas motor neuronal type 1 terminals display large boutons and are double-labeled with Dlg and HRP (white color in $A^{\prime}$ ), terminals of type 2 modulatory VUM neurons are slim and lack Dlg [throughout this figure, white curved arrows and closeups show type 2 terminals on DA1/D01 muscles; open curved arrows show type 2 terminals on DA2/D02; for terminal classification, compare Landgraf et al. (2003)]. If $U$ neurons are stalled (arrow in $B, B^{\prime}$ ), terminals of remaining ISN type 1 terminals are almost always present (asterisk in $B$ ), and VUM neurons are seen in most dorsal positions in $96 \%$ of these cases. If aCC/RP2 neurons are stalled (white arrows in $\left(-D^{\prime}\right)$, nearly all ISN neurons coarrest (Fig $\left.4 A^{\prime}, D, E\right)$, but in $30 \%$ of cases $U$ neurons escape (asterisks in $\left(-D^{\prime}\right)$; in these cases, VUM neurons are absent on dorsal muscles in $27 \%\left(C^{\prime}\right)$ and present in the remaining $73 \%\left(D^{\prime}\right) . E-F^{\prime \prime}$, Growth cones and axons of aCC/RP2 (green in $C-C^{\prime \prime}$; RN2::GFP-actin) mostly show low levels of Fasciclinll (magenta) on exposed surfaces (curved open arrows), whereas GFP-Actin expressed in $U$ neurons (green in $D-D^{\prime \prime} ; U / C Q:: G F P$-actin) is always congruent with high levels of Fasciclinll (curved white arrows). Scale bar: $A-B^{\prime}, 25 \mu \mathrm{m}$; $\left(-D^{\prime \prime}, 8 \mu \mathrm{m}\right.$.

\section{Discussion}

The molecular basis of pioneer-follower interactions can be approached through growth manipulation of pioneers

The phenomenon of pioneer neurons was first described almost a century ago (Harrison, 1910). However, insights into the underlying molecular mechanisms are sparse. Here we use an approach that allows us to study the influences of pioneers on the growth of follower neurons. We show that aCC and/or RP2 neurons represent pioneer neurons of the ISN that have strong influencing properties and are clearly distinct in their growth cone shapes from the U neurons, although both neuron groups grow at the leading front of the ISN. We show that the growth-influencing capabilities of aCC/RP2 and also the differences between pioneer and follower growth cones are in large part dependent on FasciclinII. Although aCC/RP2 neurons have a strong influence on followers ( shown by stalling experiments), they are not absolutely required (shown by ablations), but followers can instead partially compensate for loss of pioneer neurons. Our analyses of growth cone shapes suggest explanations for this phenomenon (see below).

So far, pioneer-follower interactions have mostly been investigated by experiments in which pioneer neurons were ablated and the consequences for the follower neurons and respective nerves were analyzed. Such experiments tell us about the navigational capabilities of follower neurons. However, because potential growth-influencing cues displayed by pioneer neurons are abolished together with the ablated neurons, these experiments fail to shed light on the molecular mechanisms underlying pioneer-follower interactions. Here, we used the stalling approach, which maintains the pioneer neurons but changes their growth behaviors (see also Boschert et al., 1990). Under these conditions, we can determine to which degree the informational cues displayed by pioneer neurons influence the followers. The degree of influence turned out to be very high in the case of aCC/RP2 stalls, as judged by the high frequency of coarrested ISNs. Capitalizing on this severe coarrest phenotype, we could apply Drosophila genetics and pinpoint FasciclinII as one important molecule required for pioneer-follower interactions.

Revealing support for these results came from our analyses of growth cone shapes. In contrast to the pioneering growth cones of aCC/RP2, those of the following $\mathrm{U}$ motor axons are narrower and less complex, although they grow in the same environment at the same time. Similar shape differences have been shown for pioneer and follower neurons in vertebrates (Kim et al., 1991; Bak and Fraser, 2003), and therefore it seems to be a characteristic feature of these two neuron groups. We found that, in the absence of FasciclinII, U growth cones take on shape characteristics reminiscent of pioneer growth cones. Because similar changes on follower growth cones were observed after physical ablation of pioneer neurons in vertebrates (Bak and Fraser, 2003), we conclude that the fasciclinII-mutant background represents a genetic ablation of molecules required for the pioneers' influence on navigation and shape of follower growth cones. It appears as if filopodia of follower growth cones in the presence of FasciclinII are attracted to or influenced by cues on the surfaces of aCC/RP2. In contrast, filopodia of aCC/RP2 growth cones do not seem to be particularly attracted to natural levels of FasciclinII on the surfaces of $U$ neurons, although they grow in their immediate vicinity. This clearly shows that growth cones of both neuron groups behave differently in a wild-type background. Instead of clinging to $U$ neuronal surfaces, growth cones of aCC/RP2 appear to screen for other cues in their environment. We observed a very similar behavior for $\mathrm{U}$ growth cones in a fasciclinII ${ }^{\text {eb112 }}$ mutant background, suggesting that $U$ growth cones, in the absence of FasciclinII, seem to be able to respond to the same environmental cues as aCC/RP2. This would also explain why they can reach their destination at high frequency (74\%) after aCC/RP2 ablation. This interpretation might also provide a possible explanation for the fact that aCC and $\mathrm{U}$ neurons have exchanged roles during evolution ( $U$ neurons are thought to be the pioneers of the ISN in grasshoppers) (DuLac et al., 1986). 

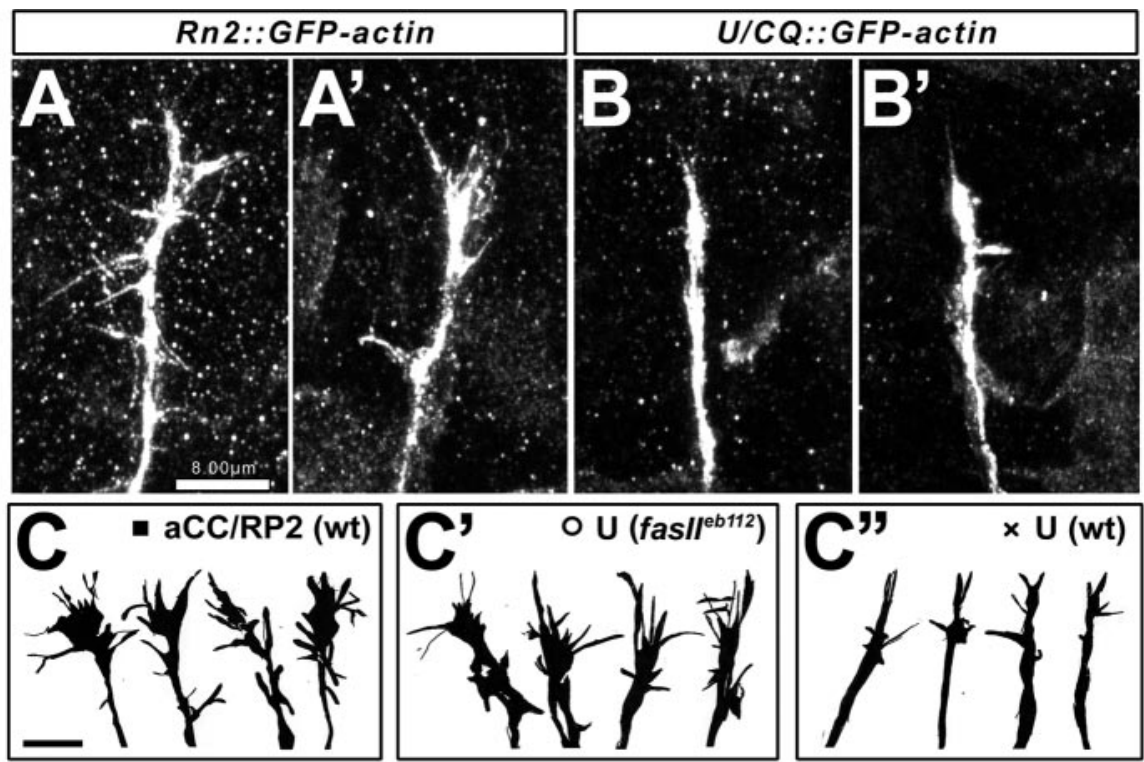

D
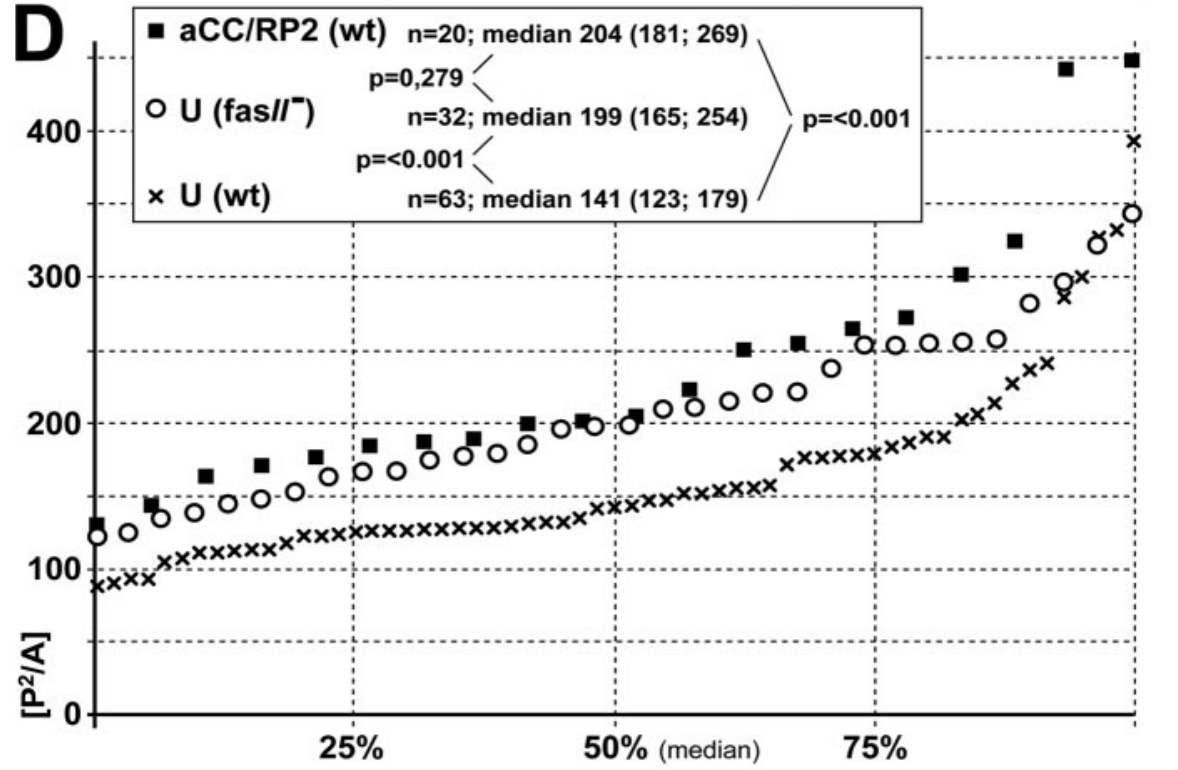

Figure 6. Shapes of growth cones distinguish pioneers from followers and are influenced by Fasciclinll. Whereas growth cones of aCC/RP2 neurons show numerous filopodia $\left(A, A^{\prime}, C\right)$, growth cones of $U$ neurons are narrow and poor in filopodia $\left(B, B^{\prime}, C^{\prime \prime}\right.$; visualized with Rn2-Gal4- or U/CQ-Gal4-driven GFP-actin, respectively). Camera lucida drawings of growth cones $\left(C-C^{\prime \prime}\right)$ were obtained from aCC/RP2 neurons in wild type (wt) (C, black square), U neurons in fasciclin/l ${ }^{\text {eb112 }}$ loss-of-function mutant background (fasll) $\left(C^{\prime}\right.$, circle), or U neurons in wild-type embryos ( $C^{\prime \prime}$, black cross). Their complexity index, $\mathrm{P}^{2} / A$, is plotted as a graph in D showing the median at $50 \%$ and interquartile ranges at 25 and $75 \%$; the box in $D$ contains sample numbers of measured growth cones $(n)$, values of the respective medians (interquartile ranges in brackets separated by a semicolon), and significance values $(p)$ obtained by Mann-Whitney rank sum tests (comparing the respective groups indicated by lines). Whereas $\mathrm{U}$ growth cones are significantly less complex than those of aCC/RP2 neurons in wild-type background, they are similarly complex in fasciclin/l ${ }^{e b 112}$ mutant background. Scale bars, $8 \mu \mathrm{m}$.

\section{The role of the N-CAM homolog FasciclinII during} pioneer-follower interaction

How does FasciclinII mediate induction of the asymmetric growth cone behaviors of pioneers and followers during normal ISN development? So far, FasciclinII and its homologs N-CAM (vertebrates) and apCAM (Aplysia) have been shown to play important roles during different kinds of neuronal growth events. They have been shown to regulate synaptic terminal size and branching in the embryo or during plastic structural reorganization at later stages, but they also play roles during axonal growth (e.g., during the processes of nerve defasciculation or target rec-

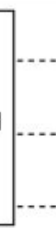

ognition) (for review, see Goodman et al., 1997; Schachner, 1997; Ronn et al., 1998; Crossin and Krushel, 2000). The function of FasciclinII during pioneer-follower interactions clearly falls into the second context.

Up to now, a potential involvement of Drosophila FasciclinII in axonal growth regulation in situ was suggested by overexpression experiments. For example, targeted expression of FasciclinII in all neurons prevents axons from leaving their nerves and growing toward their target muscles (Lin and Goodman, 1994), whereas targeted expression in muscles leads to manifestation of ectopic nerve branches on inappropriate muscles (Davis et al., 1997). Both examples suggest that FasciclinII-expressing cells become more attractive or instructive to growth cones. This is in accordance with our observation that expression of FasciclinII in stalled aCC/RP2 or U neurons increases their influence on ISN growth (Figs. 1, 4). However, targeted expression of FasciclinII might induce artificial phenotypes that do not necessarily reflect the function of endogenous protein. This counterargument could be invalidated by the demonstration that removal of endogenous protein ( fasciclinII $^{\text {ebl12 }}$ ) led to a reduction of the influence of aCC/RP2 on $U$ growth and to a spreading of $U$ growth cones. These results clearly show that endogenous FasciclinII is also required for growth-regulating pioneer-follower interactions.

There are several ways in which FasciclinII could function during pioneer-follower interactions. N-CAMs usually act through homophilic or heterophilic engagement with other molecules on adjacent cell surfaces or in the extracellular matrix. They can function as adhesion molecules, signal sending ligands, and/or signal-transducing receptors (Ronn et al., 1998; Crossin and Krushel, 2000; Suter and Forscher, 2001). We favor the hypothesis that FasciclinII in the pioneer context does not simply work as an equally bidirectional homophilic adhesion factor. Instead, we believe that it acts via signaling, as demonstrated recently in Drosophila cell culture (Forni et al., 2004). Our view is based on several arguments. First, although FasciclinII is expressed in all neurons of the ISN (Grenningloh et al., 1991; Vactor et al., 1993), aCC/RP2 can influence the later-growing FasciclinII-positive VUM neurons more efficiently than $U$ neurons. If aCC/RP2 and $U$ neurons interacted in homophilic mutual ways, both should have a similar impact. Second, our growth cone analysis suggests that $U$ growth cones attach to aCC/RP2, whereas aCC/RP2 growth cones do not seem to be particularly attracted to U neurons. Third, FasciclinII levels on exposed surfaces of aCC/RP2 but not of $U$ neurons are surprisingly low during the phase of axonal outgrowth (but the 
possibility that they are similarly enriched in both growth cones at shared contact sites cannot be excluded). These stainings were performed with an antibody detecting the intracellular domain of FasciclinII, leaving open the possibility that aCC may predominantly display the glycosylphosphatidylinositol-anchored isoform that lacks the intracellular domain (Goodman et al., 1997). In contrast, the follower neurons contain high levels of isoforms containing the intracellular domain. Therefore, an attractive hypothesis would be that intracellular signaling relevant to growth regulation is mediated by the intracellular domain of FasciclinII. This way, the follower neurons would be more sensitive to other FasciclinII-positive neuronal surfaces than aCC/RP2. Only induced overexpression of FasciclinII in follower neurons might reach levels sufficient to trigger responses in aCC/RP2, as revealed by their increased stall frequency in those experiments. Furthermore, we have to consider that, in analogy to its homologous proteins in other species, FasciclinII might also interact in a heterophilic manner or show different modes of intracellular molecular interactions in different cellular contexts (i.e., in pioneer and follower neurons). Also, we cannot determine whether FasciclinII functions as an instructive molecule during pioneer guidance or acts only in a permissive way together with other factors. The cellular system we have presented here provides the genetic and experimental possibilities to address these details in the natural context of developing embryos.

\section{References}

Bak M, Fraser SE (2003) Axon fasciculation and differences in midline kinetics between pioneer and follower axons within commissural fascicles. Development 130:4999-5008.

Bate M (1993) The mesoderm and its derivatives. In: The development of Drosophila melanogaster (Bate M, Martínez Arias A, eds), pp 1013-1090. Cold Spring Harbor, NY: Cold Spring Harbor Laboratory.

Boschert U, Ramos RG, Tix S, Technau GM, Fischbach KF (1990) Genetic and developmental analysis of irre $C$, a genetic function required for optic chiasm formation in Drosophila. J Neurogenet 6:153-171.

Bowie JE, Young IT (1977) An analysis technique for biological shape-II. Acta Cytol 21:455-464.

Brand A, Perrimon N (1993) Targeted gene expression as a means of altering cell fates and generating dominant phenotypes. Development 118:401-415.

Broadie KS (2000) Electrophysiological approaches to the neuromuscular junction. In: Drosophila protocols (Sullivan W, Ashburner M, Hawley RS, eds), pp 273-295. Cold Spring Harbor, NY: Cold Spring Harbor Laboratory.

Broadus J, Skeath JB, Spana E, Bossing T, Technau GM, Doe CQ (1995) New neuroblast markers and the origin of the aCC/pCC neurons in the Drosophila CNS. Mech Dev 54:1-10.

Crossin KL, Krushel LA (2000) Cellular signaling by neural cell adhesion molecules of the immunoglobulin superfamily. Dev Dyn 218:260-279.

Davis GW, Schuster CM, Goodman CS (1997) Genetic analysis of the mechanisms controlling target selection: target-derived Fasciclin II regulates the pattern of synapse formation. Neuron 19:561-573.

Duffy JB (2002) GAL4 system in Drosophila: a fly geneticist's Swiss army knife. Genesis 34:1-15.

DuLac S, Bastiani MJ, Goodman CS (1986) Guidance of neuronal growth cones in the grasshopper embryo. II. Recognition of a specific axonal pathway by the aCC neuron. J Neurosci 6:3532-3541.

Eisen JS, Pike SH, Debu B (1990) The growth cones of identified motoneurons in embryonic zebrafish select appropriate pathways in the absence of specific cellular interactions. Neuron 2:1097-1104.

ffrench-Constant RH (1993) Cloning of the Drosophila cyclodiene insecticide resistance gene: a novel $\mathrm{GABA}_{\mathrm{A}}$ receptor subtype? Comp Biochem Physiol 104:9-12.

Forni JJ, Romani S, Doherty P, Tear G (2004) Neuroglian and FasciclinII can promote neurite outgrowth via the FGF receptor Heartless. Mol Cell Neurosci 26:282-291.

Fujioka M, Emi-Sarker Y, Yusibova GL, Goto T, Jaynes JB (1999) Analysis of an even-skipped rescue transgene reveals both composite and discrete neuronal and early blastoderm enhancers, and multi-stripe positioning by gap gene repressor gradients. Development 126:2527-2538.

Fujioka M, Lear BC, Landgraf M, Yusibova GL, Zhou J, Riley KM, Patel NH, Jaynes JB (2003) Even-skipped, acting as a repressor, regulates axonal guidance in Drosophila. Development 130:5385-5400.

Ghosh A, Antonini A, McConnell SK, Shatz CJ (1990) Requirement for subplate neurons in the formation of thalamocortical connections. Nature 347:179-181.

Goodman CS, Davis GW, Zito K (1997) The many faces of fasciclin II: Genetic analysis reveals multiple roles for a cell adhesion molecule during the generation of neuronal specificity. Cold Spring Harb Symp Quant Biol 62:479-491.

Grenningloh G, Rehm EJ, Goodman CS (1991) Genetic analysis of growth cone guidance in Drosophila: Fasciclin II functions as a neuronal recognition molecule. Cell 57:45-57.

Harrison RG (1910) The outgrowth of the nerve fiber as a mode of protoplasmic movement. J Exp Zool 9:787-846.

Hidalgo A, Brand AH (1997) Targeted neuronal ablation: the role of pioneer neurons in guidance and fasciculation in the CNS of Drosophila. Development 124:3253-3262.

Hidalgo A, Urban J, Brand AH (1995) Targeted ablation of glia disrupts axon tract formation in the Drosophila CNS. Development 121:3703-3712.

Jacobs RJ, Goodman CS (1989) Embryonic development of axon pathways in the Drosophila CNS. II. Behaviour of pioneer growth cones. J Neurosci 9:2412-2422.

Jan Y-N, Jan L-L (1982) Antibodies to horseradish peroxidase as specific neuronal markers in Drosophila and grasshopper embryos. Proc Natl Acad Sci USA 79:2700-2704

Keshishian H, Bentley D (1983) Embryogenesis of peripheral nerve pathways in grasshopper legs. III. Development without pioneer neurons. Dev Biol 96:116-124.

Kidd S, Lieber T, Young MW (1998) Ligand-induced cleavage and regulation of nuclear entry of Notch in Drosophila melanogaster embryos. Genes Dev 12:3728-3740.

Kiehart DP, Feghali R (1986) Cytoplasmic myosin from Drosophila melanogaster. J Cell Biol 103:1517-1525.

Kim GJ, Shatz CJ, McConnell SK (1991) Morphology of pioneer and follower growth cones in the developing cerebral cortex. J Neurobiol 22:629-642.

Klagges BRE, Heimbeck G, Godenschwege TA, Hofbauer A, Pflugfelder GO, Reifegerste R, Reisch D, Schaupp M, Buchner S, Buchner E (1996) Invertebrate synapsins: a single gene codes for several isoforms in Drosophila. J Neurosci 16:3154-3165.

Klose M, Bentley D (1989) Transient pioneer neurons are essential for formation of an embryonic peripheral nerve. Science 245:982-984.

Kuwada JY (1986) Cell recognition by neuronal growth cones in a simple vertebrate embryo. Science 233:740-746.

Lahey T, Gorczyca M, Jia XX, Budnik V (1994) The Drosophila tumor suppressor gene $d l g$ is required for normal synaptic bouton structure. Neuron 13:823-835.

Landgraf M, Roy S, Prokop A, VijayRaghavan K, Bate M (1999) evenskipped determines the dorsal growth of motor axons in Drosophila. Neuron 22:43-52.

Landgraf M, Sánchez-Soriano N, Technau GM, Urban J, Prokop A (2003) Charting the Drosophila neuropile: a strategy for the standardised characterisation of genetically amenable neurites. Dev Biol 260:207-225.

Lee T, Luo L (1999) Mosaic analysis with a repressible neurotechnique cell marker for studies of gene function in neuronal morphogenesis. Neuron 22:451-461.

Lin DM, Goodman CS (1994) Ectopic and increased expression of Fasciclin II alters motoneuron growth cone guidance. Neuron 13:507-523.

Lin DM, Auld VJ, Goodman CS (1995) Targeted neuronal cell ablation in the Drosophila embryo: pathfinding by follower growth cones in the absence of pioneers. Neuron 14:707-715.

Luo L, Liao YJ, Jan LY, Jan YN (1994) Distinct morphogenetic functions of similar small GTPases: Drosophila Drac1 is involved in axonal outgrowth and myoblast fusion. Genes Dev 8:1787-1802.

Murray MJ, Merritt D, Brand AH, Whitington PM (1998) In vivo dynamics of axon pathfinding in the Drosophila CNS: a time-lapse study of an identified motor neuron. J Neurobiol 37:607-621. 
Pike SH, Melancon EF, Eisen JS (1992) Pathfinding by zebrafish motoneurons in the absence of normal pioneer axons. Development 114:825-831.

Raper JA, Bastiani MJ, Goodman CS (1984) Pathfinding by neuronal growth cones in grasshopper embryos. IV. The effects of ablating the A and $\mathrm{P}$ axons upon the behavior of the G growth cone. J Neurosci 4:2329-2345.

Ronn LC, Hartz BP, Bock E (1998) The neural cell adhesion molecule (NCAM) in development and plasticity of the nervous system. Exp Gerontol 33:853-864.

Schachner M (1997) Neural recognition molecules and synaptic plasticity. Curr Opin Cell Biol 9:627-634.

Schuster CM, Davis WD, Fetter RD, Goodman CS (1996) Genetic dissection of structural and functional components of synaptic plasticity. I. Fasciclin II controls synaptic stabilisation and growth. Neuron 17: 641-654.

Spradling AC, Rubin GM (1982) Transposition of cloned P elements into Drosophila germ line chromosomes. Science 218:341-347.

Suter DM, Forscher P (2001) Transmission of growth cone traction force through apCAM-cytoskeletal linkages is regulated by Src family tyrosine kinase activity. J Cell Biol 155:427-438.

Tessier-Lavigne M, Goodman CS (1996) The molecular biology of axon guidance. Science 274:1123-1133.

Thomas JB, Bastiani MJ, Bate M, Goodman CS (1984) From grasshopper to Drosophila: a common plan for neural development. Nature 310:203-206.

Tosney KW, Landmesser LT (1985) Growth cone morphology and trajectory in the lumbosacral region of the chick embryo. J Neurosci 5:2345-2358.

Vactor DV, Sink H, Fambrough D, Tsoo R, Goodman CS (1993) Genes that control neuromuscular specificity in Drosophila. Cell 73:1137-1153.

Verkhusha VV, Tsukita S, Oda H (1999) Actin dynamics in lamellipodia of migrating border cells in the Drosophila ovary revealed by a GFP-actin fusion protein. FEBS Lett 445:395-401.

Williams DW, Shepherd D (2002) Persistent larval sensory neurones are required for the normal development of the adult sensory afferent projections in Drosophila. Development 129:617-624.

Woods DF, Bryant PJ (1991) The discs-large tumor suppressor gene of Drosophila encodes a guanylate kinase homolog localized at septate junctions. Cell 66:451-464. 